\title{
The relation between memory and expectancy as revealed by percentage and sequence of reward investigations
}

\author{
E. J. CAPALDI \\ Purdue University, West Lafayette, Indiana
}

\begin{abstract}
There is growing agreement that to explain instrumental learning properly, one should emphasize memory as well as expectancy. I call this approach memory-expectancy theory. Amsel's (1992) frustration theory is one variety of memory-expectancy theory. Capaldi's (1994) sequential theory is another. In this report, I examine in considerable detail the effects of percentage and sequence of reward on extinction following different levels of acquisition training. These extinction findings, taken together with certain serial learning acquisition findings, seem to support a novel version of memory-expectancy theory, one that in some respects is similar to and in some respects is different from that suggested by Amsel. First, on the basis of this analysis, we may reject two ideas: that animals remember only the prior reward event and that animals anticipate only the reward event contingent upon the current response. Second, the analysis supports three salient propositions of the present memory-expectancy approach. Memories of reward events may serve as conditioned stimuli for expectancies of reward events. On any current trial, the animal may remember each of the reward events associated with one or more prior trials. On any current trial, the animal may anticipate not only the current reward event, but also reward events contingent upon subsequent trials. Essentially, according to this model, the stimuli that elicit expectancies, as well as the expectancies themselves, may change progressively over a series of learning trials.
\end{abstract}

Amsel's frustration theory is notable for at least two significant reasons. It is a logical and necessary development of the highly influential conditioning model theory, allowing it to deal successfully with a variety of new and interesting but otherwise intractable phenomena. Too, the theory has proved to be extremely fruitful. Amsel's frustration view is clearly a major theory of learning. His book (Amsel, 1992) appropriately describes many of the achievements of frustration theory. The major achievements of the theory consist in identifying the mechanisms that regulate instrumental responding under a wide variety of experimental conditions. This concern, which is currently a topic of intense investigation (e.g., Capaldi, 1994; Colwill, 1993; Rescorla, 1994), will occupy my attention here. My view of the matter is in some respects similar to, and in some respects different from, that of Amsel (see Capaldi, 1994; Capaldi, Birmingham, \& Alptekin, in press).

Conditioning model theory as it has been developed by Amsel holds that instrumental behavior is regulated extensively by stimuli produced by classically conditioned responses. In Amsel's particular version of conditioning model theory, internal classically conditioned goal responses produce stimuli that both motivate and di-

Correspondence should be addressed to E. J. Capaldi, Department of Psychology, Purdue University, 1364 Psychology Building, West Lafayette, IN 47907-1364. rect instrumental responding. These classically conditioned responses are referred to as expectancies or anticipations.

A diagram commonly employed by Amsel to represent these associations under a schedule of partial reward is shown below (see, e.g., Amsel, 1967):<smiles>[R][R]([R])([R])[Si](F)(S)[Si]F</smiles>

In this diagram, the exteroceptive and static apparatus cues $\left(S_{A}\right)$ come to elicit the anticipatory responses $r_{R}$ and $r_{F}$. The classically conditioned anticipatory reward response, $r_{R}$, produces the stimulus, $s_{R}$, and the classically conditioned anticipatory frustrative response, $\mathrm{r}_{\mathrm{F}}$, produces the stimulus $\mathrm{S}_{\mathrm{F}}$. The stimuli produced by the anticipatory responses that become conditioned to the instrumental approach response (IR) are the workhorses, so to speak, of frustration theory. It would be impossible to overestimate the importance of $\mathrm{s}_{\mathrm{R}}$ and $\mathrm{s}_{\mathrm{F}}$ within frustration theory; they are the theory's heart. If frustration theory has to do with anything, it has to do with how internal stimuli produced by various reward events come to direct and motivate instrumental responding. It is this central aspect of frustration theory with which I am concerned here.

As important as $\mathrm{s}_{\mathrm{R}}$ and $\mathrm{s}_{\mathrm{F}}$ are within frustration theory, Amsel indicates that another class of goal-related 
interoceptive stimuli also exercises control over instrumental responding. As Amsel (1994) puts it, these other stimuli arise

as a direct consequence of what happened on the last learning trial, the direct feed forward from trial $N$ to trial $N+1$, which can be characterized as a short- or immediateterm trace or memory of the last goal experience that controls the next. This has been the interoceptive stimulus mechanism featured in many of Capaldi's (e.g., 1967) explanations of reward schedule learning, and in his view these stimuli can account for relatively long-term consequences of reinforcement and nonreinforcement. (p. 15)

Note that I have attached as much importance to the memories of reward events as Amsel has to the expectancies of reward events.

In Pavlovian conditioning, as temporal conditioning illustrates, any stimulus, including interoceptive stimuli, may serve as a conditioned stimulus (CS). I have suggested recently (Capaldi, 1994; Capaldi et al., in press) that the interoceptive stimulus such as the memory of a prior reward event $\left(\mathrm{S}^{\mathrm{R}}\right)$ and the memory of a prior nonreward event $\left(\mathrm{S}^{\mathrm{N}}\right)$ may serve as CSs for the expectancies of reward $\left(E_{R}\right)$ and of nonreward $\left(E_{N}\right)$. Both memories and expectancies may come to elicit the instrumental reaction (IR). I (Capaldi, 1994; see also, Capaldi et al., in press) have indicated, in some detail, how, under a wide variety of conditions, memories of reward events are more valid signals for either reward or nonreward than are static exteroceptive stimuli such as apparatus cues $\left(\mathrm{S}_{\mathrm{A}}\right)$ - the sort of cues usually emphasized in conventional treatments of conditioning model theory (see, e.g., Amsel, 1992). I favor an extension of conditioning model theory which emphasizes that reward-produced memories may serve as CSs for expectancies of reward and nonreward. These are considered to be S-S associations rather than $\mathrm{S}-\mathrm{R}$ associations of the sort favored by Amsel (1992).

Below are shown four different associative structures as examples of what I have in mind. Each of the last three structures is capable of producing increased resistance to extinction relative to the first, because $\mathrm{S}^{\mathrm{N}}, \mathrm{E}_{\mathrm{N}}$, or both acquire control over IR in acquisition and subsequently occur in extinction. Shown in Structure 1 is that the memory of reward, $S^{R}$, elicits $E_{R}$, a Pavlovian relation, and that both $S^{R}$ and $E_{R}$ elicit IR, an instrumental relation. Structure 1 is that which would arise under a schedule of consistent reinforcement; that is, reward, or $\mathrm{R}$, is both remembered and expected on each trial, both $\mathrm{S}^{\mathrm{R} 1}$ and $\mathrm{E}_{\mathrm{R}}$ occur on $\mathrm{R}$ trials, and so both enter into an excitatory relation with IR. Structure 2 , in which the memory of nonreward $\mathrm{S}^{\mathrm{N} 1}$ elicits $\mathrm{E}_{\mathrm{R}}$ and both elicit IR, would arise under a schedule of partial reward in which each nonrewarded or $\mathrm{N}$ trial is followed by a rewarded or R trial, such as a schedule in which $\mathrm{R}$ and $\mathrm{N}$ trials alternate. Structure 3, a relatively common structure, could arise under an irregular schedule of partial reward in which $\mathrm{N}$ trials are followed by $\mathrm{N}$ trials as well as by $\mathrm{R}$ trials. Structure 4 , which was extensively examined by
Capaldi et al. (in press), arises when an $\mathrm{R}$ trial is followed by an $\mathrm{N}$ trial.

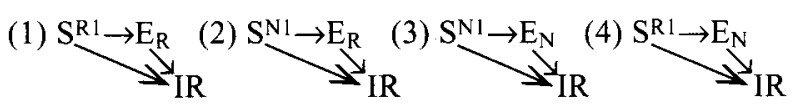

\section{The Growth of Memory-Expectancy Theory}

The view that instrumental behavior is controlled by the memory of prior reward events is an example of intertrial theory. The view that instrumental behavior is controlled by expected reward events is an example of intratrial theory. At an earlier time, intertrial and intratrial theories were seen as rival approaches in serious competition. Moreover, intratrial theories were seen as having won the competition. For example, Lawrence and Festinger (1962) said, "The major shifts in these accounts over the years has been from an emphasis on intertrial sources of stimulation, as in the Sheffield account, to a reliance on intratrial factors such as frustration responses and competing behaviors" (p. 29).

The theoretical climate has changed greatly since Lawrence and Festinger (1962) voiced their opinion about the relation between intratrial and intertrial theories. As indicated, Amsel's (1994) theory, which is primarily an intratrial theory, explicitly leaves room for an intertrial mechanism, a short-term or long-term stimulus trace or memory contributed by the previous trial (see also Amsel, 1967). Too, as indicated above, I (1994; see also Capaldi et al., in press) have recently incorporated into his intertrial theory an intratrial mechanism similar to Amsel's. In any event, it has become commonplace to explain instrumental and classical conditioning in terms of memory (see, e.g., Spear \& Riccio, 1994). Finally, a considerable variety of theorists concerned with effects other than reward schedule effects have suggested that instrumental behavior is controlled both by the memories of prior events, including memories of reward events, and by the anticipation of future events, including anticipated reward events (e.g., Chatlosh \& Wasserman, 1992; Grant, 1981; Honig \& Thompson, 1982; Linwick, Overmier, Peterson, \& Mertens, 1988). Thus, the view that we require both an intertrial memory mechanism and an intratrial anticipatory mechanism to understand instrumental behavior seems to be widely shared and growing. To be sure, there are many differences among the various versions of what I call memory-expectancy theory. Nevertheless, that so many have embraced the memory-expectancy approach to learning is itself noteworthy and perhaps significant.

\section{Current Problems With the Memory-Expectancy Approach}

The recognition by many individuals that instrumental behavior is controlled by two classes of interoceptive stimuli-memories of prior reward events and expectancies of contingent reward events - may constitute a major advance in theorizing. Nevertheless, that step, while perhaps necessary, can be expected, at least in the 
short run, to add to our difficulties rather than reduce them. It is the case that any blend of memories and expectancies-any blend whatsoever-raises a variety of as yet unresolved and largely unexamined issues. As one example, it is often the case that each mechanism explains some given phenomenon equally well; for example, some phenomena can be explained in terms of either an $\mathrm{S}^{\mathrm{N}}$-IR association alone or an $\mathrm{E}_{\mathrm{N}}-\mathrm{IR}$ association alone. Thus, it is difficult to determine, for any variety of memory-expectancy theory, whether under a given set of conditions one should emphasize the memory mechanism, the expectancy mechanism, or both. Determining the explanatory scope and limits of each mechanism will not prove to be easy. At this stage of development of memory-expectancy theory, we cannot expect definitive answers to the three issues I will raise in what follows. However, my hope is that in the raising of these issues, the first tentative step toward resolving them, as well as others, will have been taken.

\section{Three Issues}

First, the relationship between the two mechanisms might be one of independence, each mechanism exercising separate and independent control over behavior (see, e.g., Linwick et al., 1988). Or it might be, as already indicated, that the two mechanisms work in tandem with memories serving as CSs for expectancies exactly as would any other stimuli (Capaldi, 1994; Capaldi et al., in press).

Second, while it is widely agreed that expectancies arise out of classical conditioning, the stimuli produced by the intertrial mechanism have been seen as more or less rapidly fading traces (see Amsel, 1992; Grant, 1981; Sheffield, 1949) or as memories that follow the same laws as any other memories do (see, Capaldi, 1994; Capaldi et al., in press).

Finally, there is the issue of how many events animals may remember and how many they may anticipate. One view, apparently, is that on any given trial the animal remembers only the prior reward event (e.g., Amsel, 1994; Hull, 1952; Sheffield, 1949). Too, it appears to be assumed, implicitly if not explicitly, that animals anticipate only the reward event contingent upon the current response (e.g., Amsel, 1992; Chatlosh \& Wasserman, 1992; Linwick et al., 1988). An alternative view is that animals may remember one, two, or more prior reward events (and the order in which they occurred) while anticipating not only the reward event contingent upon the current response but also reward events contingent upon one or more future responses (see, e.g., Capaldi, 1985, 1992, 1994; Capaldi \& Verry, 1981). The ability of animals to remember and to anticipate multiple past and future reward events is my concern in this report.

\section{The Relation Between Expectancy and Percentage of Reward}

Examining the effects of percentage of reward on extinction provides a good initial avenue for analyzing different views concerned with the role of memories and expectancies in jointly controlling instrumental behavior. According to Amsel (1992), as the discrepancy grows between $r_{R}$ and the reward outcome actually received, the greater is the intensity of frustration conditioned to the instrumental reaction under, say, a schedule of partial reward. Some variables that should lead to bigger $r_{R}$ include increased magnitude of food reward and increased number of food-rewarded trials. What is the effect of percentage of reward on $r_{R}$ ? A definitive answer seems to be lacking within frustration theory (see, e.g., Spence, 1960). One possibility is that if enough training is given, ultimately $r_{R}$ will have the same value for all reward percentages. That is, it may simply take more trials for $\mathrm{r}_{\mathrm{R}}$ to achieve its final or asymptotic level of growth the lower the reward percentage. A second possibility is that $r_{R}$ will be weaker, the lower the reward percentage even after extended training. That is, the lower the percentage of reward, the weaker will be the expectancy of reward or $\mathrm{r}_{\mathrm{R}}$. For a more formal statement of these alternatives, see Spence (1960). I am going to suggest that, on the basis of current information, the second of the two possibilities listed above is the more feasible. First, Pavlovian studies seem to suggest that lower reward percentages produce weaker conditioned responses (see, e.g., Mackintosh, 1974). Second, instrumental findings, some of which have been mentioned elsewhere (Capaldi, 1994), seem more compatible with the second alternative. Finally, the second alternative, but not the first, is compatible with sequence of reward studies that appear to be closely related to percentages of reward studies, as will become clear later.

\section{Percentage of Reward and Extinction: \\ The Basic Findings}

The most systematic and comprehensive study of the joint effects of percentage of reward and acquisition level on extinction in animals was that reported in Bacon's (1962) dissertation conducted under the direction of Stu Hulse. Bacon combined four percentages of reward, $30 \%, 50 \%, 70 \%$, and $100 \%$, with four acquisition levels, $10,30,100$, and 300 trials, respectively. Bacon's partial reward schedules were irregular, his subjects were rats, his apparatus was a runway, his reward a $1 \%$ saccharine solution, his intertrial interval or retention interval was $1 \mathrm{~min}$, and rats received 10 trials each day in acquisition and extinction. Each group received $30 \mathrm{ex}-$ tinction trials. Unfortunately, percentage data as comprehensive as those reported by Bacon are not available for long retention intervals.

Figure 1 shows the mean running speed in extinction for each of the 16 groups employed by Bacon. Following 10 acquisition trials, resistance to extinction increased as percentage of reward increased. But following 300 acquisition trials, resistance to extinction tended to increase as percentage of reward decreased. Given the trend of the findings shown in Figure 1, it is reasonable to suppose that if Bacon had employed more than 30 extinction trials, he might have obtained at the higher training levels a difference between the $30 \%$ and $50 \%$ 




Figure 1. The effect of different numbers of acquisition trials upon mean extinction running speed as a function of percentage of reinforcement.

groups, with the $30 \%$ group being more resistant to extinction than the $50 \%$ group. In any event, 300 acquisition trials is a very considerable number of trials for a runway investigation. My working assumption will be that further training would not materially change the results obtained at the 300-trial level. That is, given the trend of the data shown in Figure 1 from 10 to 300 trials, it seems reasonable to conclude, as did Bacon, that if sufficient acquisition training is given, resistance to extinction will tend to increase as percentage or reinforcement decreases, at least under the conditions employed in his investigation.

\section{Explaining Percentage of Reward: Memory and Expectancy I}

In massed trial extinction such as that employed by Bacon (1962), it may be assumed that both memory and anticipation are jointly controlling behavior. An important assumption here is that animals will resist extinction to the extent that the memory of nonreward, the expectancy of nonreward, or both have become signals for the instrumental response in acquisition (see Capaldi, 1994; Capaldi et al., in press). Before applying a memoryexpectancy analysis to Bacon's data, it will be helpful to examine memory alone and expectancy alone. In what follows, I emphasize only partial reward groups; practically any theory can predict fast extinction in the $100 \%$ group.

I begin by applying the following memory analysis, called M1, to Bacon's (1962) data. There are three slightly different versions of $\mathrm{Ml}$. According to the first version of M1 (see especially Sheffield, 1949), (1) the animal remembers only the prior reward event; (2) memories fade rapidly; and (3) memories acquire the capacity to elicit responding by occurring on rewarded instrumen- tal trials. Versions 2 and 3 of M1, as will become clear, entertain different assumptions under $\mathrm{C}$.

According to Version 1 of M1, the memory of nonreward would acquire control over responding only when a nonrewarded trial is followed at a short retention interval by a rewarded trial- that is, in transitions from nonrewarded to rewarded trials or NR transitions. We do not have an exact idea of how many NR transitions each of Bacon's (1962) groups received, because such specific schedule or sequence information was not reported. However, we can arrive at a fairly good determination here, because Bacon reported that a constraint on his schedules was that the first and last trials of each day were rewarded. On this basis, the maximum number of NR transitions received each acquisition day would be 2 , 3 , and 4 for the $30 \%, 70 \%$, and $50 \%$ groups, respectively. We could therefore expect that, on the average, the number of NR transitions from greatest to least was $50 \%$, $70 \%$, and $30 \%$, respectively. On the basis of the number of NR transitions, then, we might expect in employing M1 that the memory of nonreward would elicit responding most strongly in the $50 \%$ group and least strongly in the $30 \%$ group, with the $70 \%$ group being intermediate. Thus, we could expect greatest to least resistance to extinction to occur in the order $50 \%, 70 \%, 30 \%$. Only following 30 acquisition trials was this prediction from M1 close to being confirmed. Thus, Version 1 of M1 fails to explain Bacon's extinction data. But there is still another reason to reject M1 in the light of Bacon's data. Following 300 acquisition trials, it may be assumed that each of the partial reward groups received sufficient NR transition to render asymptotic the capacity of the memory of nonreward to elicit the IR, and so the partial groups should not differ in extinction. Note that the memory of nonreward would acquire this asymptotic tendency even if contiguity principle rather than reinforcement principle were employed here (Version 2 of M1). We may reject Versions 1 and 2 of M1.

Version 3 of M1 assumes that the capacity of the memory of nonreward to elicit responding not only increases on rewarded trials (NR transitions) but, as well, decreases on nonrewarded trials (transitions from nonrewarded to nonrewarded trials, $\mathrm{NN}$ ). If this were the case, the capacity of the memory of nonreward to elicit responding following, say, 300 acquisition trials as were employed by Bacon would be stronger in the $70 \%$ group than in the $30 \%$ group. This is because the $30 \%$ group received many more NN transitions than did the $70 \%$ group, which tended to receive mainly NR transitions. In employing M1, then, it could be expected (contrary to Bacon's findings) (1) that, following considerable training, the partial groups would fail to differ in extinction (Versions 1 and 2), and (2) that, following considerable training, the $70 \%$ group would show greater resistance to extinction than would the $30 \%$ group (Version 3). We may reject all three versions of M1.

Granting that M1 fails to explain Bacon's (1962) extinction findings, let us turn to an expectancy alone. 
analysis, one that sees expectancies as being elicited by static apparatus cues. Recall that earlier I gave three general reasons for rejecting the view that different percentages of reward merely determine the rate of growth of $r_{R}$ to a common asymptote. Call that view of expectancy E1. Consider a particular example of the second of the two general reasons previously given for rejecting El. If El were correct, the following would be true of all partial reward groups such as the $30 \%, 50 \%$, and $70 \%$ groups employed by Bacon. If enough acquisition training were given, eventually all would have the same $r_{R}$ and the same $r_{F}$, and all would be equally resistant to extinction. This, of course, is contrary to the trend of results reported by Bacon. We may reject E1.

Consider, now, the view that as the percentage of reward increases, $r_{R}$ increases, and, thus, the intensity of $\mathrm{s}_{\mathrm{F}}$ conditioned to the instrumental response increases. Call this view E2. A difficulty for E2 is the following. Under partial reward, resistance to extinction is greater for large reward than for small reward (e.g., Wagner, 1961). This finding has been explained in terms of the greater intensity of frustration conditioned to the reaction under the larger magnitude of partial reward (see, e.g., Amsel, 1962; Wagner, 1963). On this basis, we would expect higher percentages of partial reward to produce greater resistance to extinction than lower percentages, provided sufficient acquisition training is given. It may be that, as Amsel's theory is formulated, similar arguments apply to increased magnitudes and percentages of partial reward. One way to avoid this, perhaps, is to assume that larger rewards produce stronger conditioning, something Amsel has yet to comment on, apparently.

Perhaps there is a way to square the reward magnitude and percentage data with E2, a way that Amsel has commented on. There is a case - that in which partial reward is given after consistent reward-in which Amsel (1992) suggests that having weaker frustration conditioned to the instrumental reaction will produce increased resistance to extinction. According to that view, weaker conditioned frustration may support responding in the latter extinction trials, where frustration is less intense because of declining $r_{R}$. Perhaps this line of reasoning could be extended to the percentage case. That is, in the later extinction trials, the less intense frustration better conditioned to the reaction under the lower percentages in acquisition might sustain responding. However, if this approach is applicable to percentage of partial reward, why is it not also applicable to smaller magnitudes of partial reward, which should also produce greater resistance to extinction? An additional, perhaps better, reason for rejecting this line of argument will be suggested later when sequence of reward is examined.

Amsel might suggest that E2 has problems with Bacon's (1962) data because in that investigation the retention interval was short and thus M1 also must be considered. Perhaps this is so. However, I am inclined to doubt that any combination of M1 and E2 (or M1 and E1) will explain Bacon's data. Thus, it may be that frus- tration theory as currently formulated by Amsel (1992) may be unable to explain Bacon's findings.

\section{Explaining Percentage of Reward: Memory and Expectancy II}

There is a memory analysis, call it M2, that can explain Bacon's data and much more besides. M2 differs from Version 3 of M1 in one major respect. It assumes that the animal may not only remember nonreward from the immediately previous trial but also remember nonrewards associated with two, three, or more prior trials. Label the memories associated with, say, one, two, and three successively nonrewarded trials as $\mathrm{S}^{\mathrm{N} 1}, \mathrm{~S}^{\mathrm{N} 2}, \mathrm{~S}^{\mathrm{N} 3}$, etc. It is perhaps clear that, all else being equal, a memory occurring late in extinction, say, $\mathrm{S}^{\mathrm{N} 20}$, will receive a greater generalized capacity to elicit responding from the more similar $\mathrm{S}^{\mathrm{N} 3}$ than from the less similar $\mathrm{S}^{\mathrm{N} 1}$. This $N$-length approach, $N$-length being the number of consecutive nonrewarded trials preceding a rewarded trial, explains Bacon's findings rather easily. From what was said previously about the constraints that Bacon placed on his irregular partial reward schedules, it is perhaps clear that $N$-lengths could have been and probably were longer on the average in the $30 \%$ group (maximum $\mathrm{N}$-length of 7) than in the $50 \%$ group (maximum $\mathrm{N}$-length of 5) and longer in the $50 \%$ group than in the $70 \%$ group (maximum $N$-length of 3 ). Moreover, following considerable acquisition training, most or all of the $N$-lengths in each group would have acquired an asymptotic tendency to promote responding. Thus, on the basis of M2, it may be expected that following sufficient acquisition training, resistance to extinction should increase as percentage of reward decreases, at least under the experimental conditions employed by Bacon.

According to Version 3 of M2, the capacity of the memory of nonreward to elicit responding should be weakened in $\mathrm{NN}$ transitions. In the $30 \%$ group, then, would not the mainly nonrewarded lower values of $\mathrm{S}^{\mathrm{N}}$ (e.g., $\mathrm{S}^{\mathrm{N} 1}$ ) reduce, via stimulus generalization, the capacity to elicit responding of the mainly higher rewarded values of $S^{\mathrm{N}}$ (e.g., $\left.\mathrm{S}^{\mathrm{N} 5}\right)$ ? This tendency would be small and so should not reduce resistance to extinction greatly. I assume that the capacity of a nonrewarded trial to reduce the vigor of responding is an increasing function of how strongly reward is expected. This expectancy, as indicated, should be lower the lower the reward percentage. In the $30 \%$ group, the short $N$-lengths would have been infrequently rewarded and so should have produced minimal inhibition.

\section{Sequence of Reward}

By a sequence of reward study, I mean one in which the order or sequence of rewarded and nonrewarded trials is systematically and deliberately manipulated. In sequence studies from my laboratory, it has been shown that with the percentage of reward held constant, resistance to extinction increases as $\mathrm{N}$-length increases, provided that considerable acquisition training is given (see, e.g., Capaldi, 1994). If limited acquisition training 
is given, greater resistance to extinction may be associated with shorter $N$-length groups. This is because in the shorter $N$-length groups there were more NR transitions. Thus, the memory, for example, of $\mathrm{S}^{\mathrm{N} 1}$ occurred much more frequently on rewarded trials in the shorter $\mathrm{N}$ length groups than did the memory of, say, $\mathrm{S}^{\mathrm{N} 3}$ in the longer $N$-length groups. Following limited acquisition training, then, the greater associative capacity of, say, $\mathrm{S}^{\mathrm{N} 1}$ relative to $\mathrm{S}^{\mathrm{N} 3}$ may be the more decisive factor in determining resistance to extinction. But as acquisition training progresses, all memories associated with the longest $\mathrm{N}$-lengths acquire an asymptotic capacity to promote responding, and thus $\mathrm{N}$-length must be the decisive factor in determining extinction. Note that, following only 30 acquisition trials, a limited number, Bacon's $50 \%$ group (which probably received a greater number of NR transitions than the $30 \%$ and $70 \%$ group), showed slightly greater resistance to extinction than did either the $30 \%$ group or the $70 \%$ group, which is consistent with findings obtained in limited acquisition training sequences studies. In Bacon's 10-trial group, which received very limited acquisition training indeed, resistance to extinction was probably determined by the sheer number of rewarded trials in acquisition, as Bacon himself indicated. Some of the results obtained in sequence studies at short retention intervals have also been obtained at long retention intervals (see, e.g., Capaldi, 1994; Jobe \& Mellgren, 1974; Jobe, Mellgren, Feinberg, Littlejohn, \& Rigby, 1977). These studies, and others, indicate that memories of reward events may control behavior at long retention intervals (see, e.g., Capaldi, 1994).

It is perhaps clear that Bacon's (1962) extinction findings for percentage of reward and number of acquisition trials are entirely consistent with findings from my laboratory and other laboratories for sequence of reward and number of acquisition trials. That is, both sorts of extinction findings can be understood on the basis of number of NR transitions and $N$-length. If percentage of reward, as manipulated by Bacon, and sequence of reward, as manipulated by me and others, are as closely related as I think, then even more formidable problems exist for memory theory M1 and expectancy alone theories E1 and E2. As one example, it would not be possible to appeal to the assumption outlined earlier that the less intense frustration associated with lower reward percentages might sustain responding in those groups later in extinction, where frustration is less intense. Such an assumption is not applicable to the sequence studies, because in those considered, percentage of reward was held constant. Note, too, that it has been reported that a higher percentage group that had longer $N$-lengths than a lower percentage group showed the greater resistance to extinction (see Capaldi, 1994). Thus, the relationship between reward percentage and extinction is relatively weak.

\section{Expectancy Theory E3}

I accept a good deal of what Amsel has said about expectancy and what happens when expectancies are vio- lated (see, e.g., Capaldi, 1994). However, I entertain some other assumptions about expectancy, or memoryexpectancy theory if you will, that Amsel may or may not find congenial. Thus, I shall call the version of expectancy outlined here E3.

The percentage and sequence of reward extinction investigations considered earlier are consistent with the inference that animals can remember more than one prior reward event and, moreover, that they are able to do so over long retention intervals (see, e.g., Capaldi, 1992, 1994). The proposition that animals can remember one, two, three, or more prior reward events is also supported by explicit counting investigations. In those investigations, rats have responded discriminatively on the basis of the number of prior rewarded or nonrewarded events (e.g., Capaldi, 1993; Capaldi \& Miller, 1988a).

On the basis of the findings cited above and of others (see Capaldi, 1994; Capaldi et al., in press), I have suggested that memories of reward events can function as CSs for expectancies exactly as do any other stimuli. This is called the tandem hypothesis. As indicated earlier, in many instances the memories of past reward events are more valid signals for current and future reward events than are exteroceptive stimuli that are usually emphasized as CSs. On the basis of validity, then, memories would acquire greater control over expectancies than would the exteroceptive apparatus cues. As merely one example, under a single alternation schedule of rewarded and nonrewarded trials, the memories of reward and nonreward are perfectly valid signals for nonreward and reward, respectively. However, exteroceptive apparatus cues occur on both rewarded and nonrewarded trials and so are less valid cues than the memories.

Capaldi and Verry (1981) reported serial learning acquisition findings, subsequently confirmed under a variety of different conditions (see, e.g., Capaldi \& Miller, 1988b; Capaldi, Nawrocki, \& Verry, 1983) that are consistent with the extinction findings described earlier and that support E3 as well as M2. As one example, in their Experiment 5, rats received each day in a runway two different series of five trials each. One series consisted of four nonrewarded trials followed by a rewarded trial, symbolized as NNNNR; the other series consisted of a rewarded trial followed by four nonrewarded trials, RNNNN. Trials of a series were separated by about $30 \mathrm{sec}$; series, by about $15-20 \mathrm{~min}$. The two series were presented irregularly, so that the rat had no basis for correctly anticipating Trial 1 of each series. Thus, the rats ran rapidly on Trial 1 in both series. On every other trial of both series, however, with training the rats came to correctly anticipate reward and nonreward, a matter especially clear by the end of training. By correct anticipation, I mean slower running on nonrewarded than on rewarded trials. Relatively early in training, the rats ran faster in the NNNNR series than on the same numbered trials in the RNNNN series. For example, the rats ran faster on Trial 3 of the NNNNR series than on Trial 3 of the RNNNN series. Moreover, speeds increased pro gressively over Trials 2,3,4, and 5 in all later blocks in 
the NNNNR series. This tendency also occurred in the RNNNN series in earlier blocks but abated considerably by the end of training.

These findings suggest that the rats remembered each prior event in each series (M2) while anticipating not only the current reward outcome but also future reward outcomes (E3). Consider, for example, Trial 3, which in both series followed and preceded a nonrewarded trial. It appears reasonable to suppose that on Trial 3 the rats ran faster in the NNNNR series than in the RNNNN series for the following reason. While the rats were correctly anticipating nonreward on Trial 3 in both series by remembering the prior two reward outcomes in each series (thus explaining the relatively slow running on Trial 3 in both series), they were simultaneously anticipating reward on Trial 5 more strongly in the NNNNR series than in the RNNNN series. Thus, running was faster on Trial 3 of the NNNNR series than in the RNNNN series. Generally speaking, the findings under consideration can be taken to suggest that on each of the Trials 2, 3, 4, and 5 of each series, the rats were simultaneously aware of past, current, and future reward outcomes. A variety of alternative descriptions of these findings were examined and rejected by Capaldi (1985).

I suggest the following specific interpretation. Over trials, each memory in each series became progressively more similar to the terminal memory in each series, the memory occurring on Trial 5. In the NNNNR series, the terminal memory was that of four prior nonrewarded trials, $\mathrm{S}^{\mathrm{N} 4}$. In the RNNNN series, the terminal memory was that of a rewarded trial, $\mathrm{S}^{\mathrm{R}}$, and three prior nonrewarded trials, $\mathrm{S}^{\mathrm{N} 3}$. The terminal memory $\mathrm{S}^{\mathrm{N} 4}$ came to elicit the expectancy of reward, $\mathrm{S}^{\mathrm{N} 4} \rightarrow \mathrm{E}_{\mathrm{R}}$, so running was fast on Trial 5 of the NNNNR series. On the terminal trial, the memory $S^{R}$ and $S^{N 3}$ came to elicit the expectancy of nonreward, $S^{R}$ and $S^{N 3} \rightarrow E_{N}$, so running was slow on Trial 5 of the RNNNN series.

Given this view, it can be seen why, late in training, the speed of running increased progressively over trials of the NNNNR series but not the RNNNN series. In the NNNNR series, each of the memories, $\mathrm{S}^{\mathrm{N} 1}, \mathrm{~S}^{\mathrm{N} 2}$, and $\mathrm{S}^{\mathrm{N}}$, would occur on an $\mathrm{N}$ trial and so each would directly elicit the expectancy $E_{N}$. But to the extent that each of these memories is similar to $\mathrm{S}^{\mathrm{N} 4}$, there would be a generalized tendency to elicit $E_{R}$. Of course, the generalized tendency to elicit $E_{R}$ would be stronger for $S^{N 3}$ than for $\mathrm{S}^{\mathrm{N} 2}$ and stronger for $\mathrm{S}^{\mathrm{N} 2}$ than for $\mathrm{S}^{\mathrm{N} 1}$. In the RNNNN series, all memories of prior reward events occur on $\mathrm{N}$ trials and so all directly elicit $E_{N}$ and none elicits $E_{R}$. Thus, running would be slow on Trials $2,3,4$, and 5 of the RNNNN series.

Frustration theory, as developed by Amsel, has been and should continue to be a major and valuable theory of learning. However, in my view, a fundamental issue for frustration theory, and for other theories, as we go forward is this: What variety of memory-expectancy theory will prove best able to explain a wide variety of instrumental learning phenomena? According to the view outlined here, memories of reward events change progressively over a series of instrumental trials, and each memory elicits an expectancy appropriate to the reward schedule associated with that memory.

\section{REFERENCES}

AMSEL, A. (1962). Frustrative nonreward in partial reinforcement and discrimination learning. Psychological Review, 69, 306-328.

AMSEL, A. (1967). Partial reinforcement effects on vigor and persistence. In K. W. Spence \& J. T. Spence (Eds.), The psychology of learning and motivation (Vol. 1, pp. 1-65). New York: Academic Press.

AMSEL, A. (1992). Frustration theory: An analysis of dispositional learning and memory. New York: Cambridge University Press.

Amsel, A. (1994). Précis of Frustration theory: An analysis of dispositional learning and memory. Psychonomic Bulletin \& Review, 1, 280-296.

BACON, W. E. (1962). Partial-reinforcement extinction effect following different amounts of training. Journal of Comparative \& Physiological Psychology, 55, 998-1003.

Capaldi, E. J. (1967). A sequential hypothesis of instrumental learning. In K. W. Spence \& J. T. Spence (Eds.), The psychology of learning and motivation (Vol. 1, pp. 67-156). New York: Academic Press.

CaPaldi, E. J. (1985). Anticipation and remote associations: A configural approach. Journal of Experimental Psychology: Learning, Memory, \& Cognition, 11, 444-449.

CAPALDI, E. J. (1992). Levels of organized behavior in rats. In W. K. Honig \& G. Fetterman (Eds.), Cognitive aspects of stimulus control (pp. 385-404). Hillsdale, NJ: Erlbaum.

CaPAldi, E. J. (1993). Animal number abilities: Implications for a hierarchical approach to instrumental learning. In S. T. Boysen \& E. J. Capaldi (Eds.), The development of numerical competence: Animal and human models (pp. 191-209). Hillsdale, NJ: Erlbaum.

CAPAldi, E. J. (1994). The sequential view: From rapidly fading stimulus traces to the organization of memory and the abstract concept of number. Psychonomic Bulletin \& Review, 1, 156-181.

Capaldi, E. J., Birmingham, K. M., \& Alptekin, S. (in press). Memories of reward events and expectancies of reward events may work in tandem. Animal Learning \& Behavior.

Capaldi, E. J., \& Miller, D. J. (1988a). Counting in rats: Its functional significance and the independent cognitive processes that constitute it. Journal of Experimental Psychology: Animal Behavior Processes, 14, 3-17.

Capaldi, E. J., \& Miller, D. J. (1988b). The rat's simultaneous anticipation of remote events and current events can be sustained by event memories alone. Animal Learning \& Behavior, 16, 1-7.

Capaldi, E. J., Nawrocki, T. M., \& Verry, D. R. (1983). The nature of anticipation: An inter- and intraevent process. Animal Learning \& Behavior, 11, 193-198.

CAPAldi, E. J., \& VERRY, D. R. (1981). Serial order anticipation learning in rats: Memory for multiple hedonic events and their order. $A n$ imal Learning \& Behavior, 9, 441-453.

Chatlosh, D. L., \& Wasserman, E. A. (1992). Memory and expectancy in delayed discrimination procedures. In I. Gormezano \& E. A. Wasserman (Eds.), Learning and memory: The behavioral and biological substrates (pp. 61-79). Hillsdale, NJ: Erlbaum.

ColwILl, R. M. (1993). An associative analysis of instrumental learning. Current Directions in Psychological Science, 2, 111-116.

GRANT, D. S. (1981). Short-term memory in the pigeon. In N. E. Spear \& R. R. Miller (Eds.), Information processing in animals: Memory mechanisms (pp. 227-253). Hillsdale, NJ: Erlbaum.

Honig, W. K., \& ThOMPson, R. K. (1982). Retrospective and prospective processing in animal working memory. In G. H. Bower (Ed.) The psychology of learning and motivation (Vol. 16, pp. 239-283). New York: Academic Press.

Hull, C. L. (1952). A behavior system: An introduction to behavior theory concerning the individual organism. New Haven, CT: Yale University Press.

Jobe, J. B., \& Mellgren, R. L. (1974). Successive nonreinforcements (N-length) and resistance to extinction at spaced trials. Journal of Experimental Psychology, 103, 652-657. 
Jobe, J. B.. Mellgren, R. L., Feinberg, R. A., Littlejohn, R. L., \& RIGBY, R. L. (1977). Patterning, partial reinforcement, and N-length as a function of reinstatement of retrieval cues. Learning \& Motivation, 8, 77-97.

LaWrenCE, D. H., \& Festinger, L. (1962). Deterrents and reinforcement. Stanford. CA: Stanford University Press.

Linwick, D., Overmier, J. B., Peterson, G. B., \& Mertens, M. (1988). Interaction of memories and expectancies as mediators of choice behavior. American Journal of Psychology, 101, 313-334.

Markintosh, N. J. (1974). The psychology of animal learning. London: Academic Press.

RESCORLA, R. A. (1994). Transfer of instrumental control mediated by devalued outcome. Animal Learning \& Behavior, 22, 27-33.

SHFFFIELD, V. F. (1949). Extinction as a function of partial reinforce- ment and distribution of practice. Journal of Experimental Psychology, 39, $511-526$.

Spear, N. E., \& Riccio, D. C. (1994). Memory: Phenomena and principles. Boston: Allyn \& Bacon.

SPENCE, K. W. (1960). Behavior theory and learning. Englewood Cliffs, NJ: Prentice-Hall.

WAGNER, A. R. (1961). Effects of amount and percentage of reinforcement and number of acquisition trials on conditioning and extinction. Journal of Experimental Psychology, 62, 234-242.

Wagner, A. R. (1963). Overtraining and frustration. Psychological Reports, 13, 717-718.

(Manuscript received January 12, 1994; revision accepted for publication March 31, 1994.) 